\title{
MOVING CURVES AND SESHADRI CONSTANTS
}

\author{
Andreas Leopold Knutsen, Wioletta Syzdek, and Tomasz Szemberg
}

\begin{abstract}
We study families of curves covering a projective surface and give lower bounds on the self-intersection of the members of such families, improving results of EinLazarsfeld and $\mathrm{Xu}$. We apply the obtained inequalities to get new insights on Seshadri constants and geometry of surfaces.
\end{abstract}

\section{Introduction}

Throughout this paper we work over the field $\mathbb{C}$ of complex numbers.

Let $S$ be a smooth projective surface and $\mathcal{C}_{U}=\left\{\left(C_{u}, x_{u}\right) \mid u \in U\right\}$ be a nontrivial family of pointed irreducible and reduced curves in $S$ such that mult $_{x_{u}} C_{u} \geq m$ for some integer $m \geq 1$.

Ein and Lazarsfeld showed in [4], that the self-intersection of each member of the family is bounded from below

$$
C_{u}^{2} \geq m(m-1)
$$

For $m=1$ this recovers a well known fact, that a curve with negative self-intersection cannot move in a family.

For $m \geq 2 \mathrm{Xu}$ [14, Lemma 1] gives a better bound of

$$
C_{u}^{2} \geq m(m-1)+1
$$

Recall that the gonality $\operatorname{gon}(X)$ of a smooth curve $X$ is defined as the minimal degree of a covering $X \longrightarrow \mathbb{P}^{1}$. Our new bound is the following:

Theorem A. Let $S$ be a smooth projective surface. Suppose $\mathcal{C}_{U}=\left\{\left(C_{u}, x_{u}\right) \mid u \in U\right\}$ is a family of pointed curves as above parametrized by a 2-dimensional subset $U \subset$ $\operatorname{Hilb}(S)$ and $C$ is a general member of this family. Let $\widetilde{C}$ be its normalization. Then

$$
C^{2} \geq m(m-1)+\operatorname{gon}(\widetilde{C}) .
$$

The assumption that $U$ is 2-dimensional is of course essential as there are surfaces fibred by curves of arbitrarily high gonality, which have self-intersection 0 . Note, that implicitly this assumption is made also in [14, Lemma 1], as a reduced curve is singular only in a finite number of points.

An important point is that gon $(\widetilde{C})$ can be bounded below in terms of the geometry of the surface. We will do this in Lemma 3.2 below. Note for instance that clearly $\operatorname{gon}(\widetilde{C}) \geq 2$ if $S$ is irrational, so that this already improves Xu's bound.

Bounds as in Theorem A lead to interesting geometrical constrains on Seshadri constants on surfaces. We show in this direction the following result, which generalizes [12, Theorem 3.2]. The precise definition of the integer $\mu_{S}$ appearing in the statement is given in (9) in section 5. For instance, $\mu_{S} \geq 2$ if $S$ is irrational and

Received by the editors August 26, 2008. 
$\mu_{S} \geq 3$ if $\left|K_{S}\right|$ is birational.

Note. After this work was completed, we learned that Theorem A was proved independently by Bastianelli. [2]

Theorem B. Let $S$ be a smooth projective surface and $L$ a big and nef line bundle on $S$ such that for all $x \in S$

$$
\varepsilon(L ; x)<\sqrt{\left(1-\frac{1}{4 \mu_{S}}\right)} \cdot \sqrt{L^{2}} .
$$

Then $S$ is fibered by Seshadri curves.

\section{Deformation theory and self-intersection of moving curves}

In this section we will prove Theorem A.

Let $C \subset S$ be a reduced and irreducible curve on a smooth surface. Let $p_{a}=$ $h^{1}\left(\mathcal{O}_{C}\right)$ denote the arithmetic genus of $C$. Let $\widetilde{C}$ be the normalization of $C$. Then $p_{g}=h^{1}\left(\mathcal{O}_{\widetilde{C}}\right)=h^{0}\left(\omega_{\widetilde{C}}\right)$ is the geometric genus of $C$.

We have an exact sequence of coherent sheaves on $S$ (cf. [11, (1.4)])

$$
0 \longrightarrow \mathcal{T}_{C} \longrightarrow \mathcal{T}_{S \mid C} \longrightarrow \mathcal{N}_{C / S}^{\prime} \longrightarrow 0,
$$

defining the equisingular normal sheaf $\mathcal{N}_{C / S}^{\prime}$ of $C$ in $S$. Its sections parametrize first-order deformations of $C$ in $S$ that are locally trivial [11, $\S 4.7 .1$ ], or equisingular.

Let $f: \widetilde{C} \rightarrow S$ be the natural morphism from the normalization of $C$ to $S$. Then we have a short exact sequence

$$
0 \longrightarrow \mathcal{T}_{\widetilde{C}} \stackrel{d f}{\longrightarrow} f^{*} \mathcal{T}_{S} \longrightarrow \mathcal{N}_{f} \longrightarrow 0,
$$

defining the normal sheaf $\mathcal{N}_{f}$ to $f$ (cf. $[11, \S 3.4 .3]$ ). Its sections parametrize firstorder deformations of the morphism $f$, that is, first-order deformations of $C$ that are equigeneric (i.e., of constant geometric genus).

Let $T \subset \mathcal{N}_{f}$ be the torsion subsheaf and let $\overline{\mathcal{N}}_{f}:=\mathcal{N}_{f} / T$. Then $\overline{\mathcal{N}}_{f}$ is an invertible sheaf on $\widetilde{C}$ and we have $\mathcal{N}_{f} \simeq \overline{\mathcal{N}}_{f} \oplus T$.

On $C$ we also have a natural exact sequence

$$
0 \longrightarrow \mathcal{O}_{C} \longrightarrow f_{*} \mathcal{O}_{\widetilde{C}} \longrightarrow \tau \longrightarrow 0,
$$

where $\tau$ is a torsion sheaf supported on $\operatorname{Sing} C$. This yields an exact sequence

$$
0 \longrightarrow \mathcal{N}_{C / S}^{\prime} \longrightarrow f_{*} \overline{\mathcal{N}}_{f} \longrightarrow \mathcal{N}_{C / S}^{\prime} \otimes \tau \longrightarrow 0
$$

(cf. $[11,(3.53)])$. It follows that

$$
h^{0}\left(\mathcal{N}_{C / S}^{\prime}\right) \leq h^{0}\left(\overline{\mathcal{N}}_{f}\right) \leq h^{0}\left(\mathcal{N}_{f}\right)
$$

Geometrically, this means that the first-order equisingular deformations are part of the first-order equigeneric deformations.

Now we are in the position to prove a slightly more precise version of Theorem A. 
Theorem 2.1. Let $\mathcal{C}_{U}=\left\{\left(C_{u}, x_{u}\right) \mid u \in U\right\}$, where $U \subset \operatorname{Hilb}(S)$, be a two-dimensional irreducible flat family of pointed, reduced and irreducible curves on a smooth projective surface $S$ such that mult $_{x_{u}} C_{u} \geq m$ for all $u \in U$ and such that the gonality of the normalization of the general curve is $\ell$. Then

$$
C_{u}^{2} \geq m(m-1)+\ell .
$$

Moreover, if equality holds, then, for general $u \in U, C_{u}$ is smooth outside $x_{u}$, and has an ordinary $m$-tuple point at $x_{u}$.

Proof. Let $C$ be a general member of the family and let $x$ be the marked point on $C$. Let $\widetilde{C}$ be the normalization of $C$ and let $f: \widetilde{C} \rightarrow S$ be the natural morphism. From (2) and the definition of $\overline{\mathcal{N}}_{f}$ above we have

$$
p_{g}(C)=1+\frac{1}{2}\left(K_{S} \cdot C+\operatorname{deg}\left(\overline{\mathcal{N}_{f}}\right)+\operatorname{length} T\right) .
$$

Since a point of multiplicity $m$ causes the geometric genus of an irreducible curve to drop at least by $\left(\begin{array}{c}m \\ 2\end{array}\right)$ with respect to the arithmetic genus, we must have

$$
p_{a}(C) \geq\left(\begin{array}{c}
m \\
2
\end{array}\right)+p_{g}(C)=\frac{1}{2} m(m-1)+p_{g}(C),
$$

so that

$$
C^{2}=2\left(p_{a}(C)-1\right)-K_{S} \cdot C \geq m(m-1)+2\left(p_{g}(C)-1\right)-K_{S} \cdot C .
$$

By our assumptions, $h^{0}\left(\mathcal{N}_{C / S}^{\prime}\right) \geq 2$, so that $h^{0}\left(\overline{\mathcal{N}}_{f}\right) \geq 2$ by (3). Considering then the morphism $C \longrightarrow \mathbb{P}^{1}$ defined by a 2-dimensional linear subspace of $H^{0}\left(\mathcal{N}_{f}\right)$, one has $\operatorname{deg}\left(\overline{\mathcal{N}_{f}}\right) \geq \ell$. Combining (5) and (7) we thus obtain

$C^{2}=2\left(p_{a}(C)-p_{g}(C)\right)+\operatorname{deg}\left(\overline{\mathcal{N}}_{f}\right)+$ length $T \geq m(m-1)+\operatorname{deg}\left(\overline{\mathcal{N}}_{f}\right) \geq m(m-1)+\ell$.

This proves (4). If equality holds in (4), then clearly we must have length $T=0$ in (5) and equality in (6) and the last statement follows.

In particular, we immediately see that $C_{u}^{2} \geq m(m-1)+2$ if $S$ is irrational.

Remark 2.2. If $K_{S} \cdot C_{u}<0$, then from (7) one also obtains that either

(i) $C_{u}^{2} \geq m(m-1)-K_{S} \cdot C_{u}$; or

(ii) $C_{u}^{2}=m(m-1)-K_{S} \cdot C_{u}-2, p_{g}\left(C_{u}\right)=0$ (whence $S$ is rational) and $C_{u}$ is smooth outside $x_{u}$.

In certain cases, as for instance in Example 4.1 below, this may give a better bound than (4).

\section{Positivity of the canonical divisor and gonality}

The next result gives lower bounds on $\ell$ in terms of the geometry of $S$.

We recall the following definition made in $[6]$ : 
Definition 3.1. Let $L$ be a line bundle on a smooth projective variety $X$. Then $L$ is birationally $k$-very ample if there is a Zariski-open dense subset $U \subseteq X$ such that, for any 0-dimensional scheme $Z$ of length $k+1$ with $\operatorname{Supp} Z \subset U$, the natural restriction map

$$
H^{0}(L) \rightarrow H^{0}\left(L \otimes \mathcal{O}_{Z}\right)
$$

is surjective.

For instance, $L$ is birationally 0 -very ample if and only if it has a section and 1very ample if and only if the rational map $\varphi_{L}$ determined by $|L|$ is birational onto its image.

We note that the notion of birational $k$-very ampleness is the "birational version" of the ordinary notion of $k$-very ampleness, in the sense that if $X^{\prime} \rightarrow X$ is a birational morphism between smooth projective varieties and $L$ is a line bundle on $X$, then $L$ is birationally $k$-very ample if and only if $f^{*} L$ is.

Lemma 3.2. Let $U \subset \operatorname{Hilb}(S)$ be a reduced and irreducible scheme parametrizing a flat family of reduced and irreducible curves on a smooth projective surface $S$ such that the gonality of the normalization of the general curve is $\ell$.

(a) If $\operatorname{dim} U \geq 1$ and $K_{S}$ is birationally $k$-very ample, then $\ell \geq k+2$.

(b) If $\operatorname{dim} U \geq 2$ and $S$ is birational to a surface admitting a surjective morphism onto a smooth curve $B$ of gonality $b$, then $\ell \geq b$.

Proof. To prove (a), pick a 1-dimensional reduced and irreducible subscheme $U^{\prime}$ of $U$. After compactifying and resolving the singularities of the universal family over $U^{\prime}$, we obtain a smooth surface $T$, fibered over a smooth curve, with general fiber $F$ a smooth curve of gonality $\ell$, and a surjective morphism $f: T \rightarrow S$. By adjunction $K_{T}$ fails to be $(\ell-1)$-very ample on the general fiber $F$. More precisely, on the general $F$, there is a one-dimensional family of schemes $\{Z\}$ of length $\ell$ such that the evaluation map

$$
H^{0}\left(K_{T}\right) \rightarrow H^{0}\left(K_{T} \otimes \mathcal{O}_{Z}\right)
$$

is not surjective. Since $K_{T}=f^{*} K_{S}+R$, where $R$ is the (effective) discriminant divisor of $f$, and $f$ is generically $1: 1$ on the fibers, we see that $K_{S}$ fails to be birationally $(\ell-1)$-very ample. Hence, $k \leq \ell-2$.

As for (b), there is by assumption a birational morphism from a smooth surface $\widetilde{S}$ to $S$ such that there is a surjective morphism $\widetilde{S} \rightarrow B$. Of course, also $\widetilde{S}$ is dominated by a two-dimensional nontrivial family of reduced and irreducible curves having normalizations of gonality $\ell$. A general such curve must dominate $B$, whence so does its normalization, so that $\ell \geq b$.

\section{Examples}

We give here a few examples involving Theorem 2.1 and Lemma 3.2.

Example 4.1. (Cubic surfaces and $\mathbb{P}^{2}$ ) We consider a smooth cubic surface $S$ in $\mathbb{P}^{3}$ and families of hyperplane sections satisfying the conditions in Theorem 2.1. Then $C_{u} \cdot K_{S}=-3$ and $p_{a}\left(C_{u}\right)=1$. Now Remark 2.2 yields that either $C_{u}^{2} \geq m(m-1)+3$ or $C_{u}^{2}=m(m-1)+1, C_{u}$ is rational and smooth outside $x_{u}$ and $x_{u}$ is a node. 
The first case happens with $m=1$ for the family of all hyperplane sections and the second indeed happens for the 2-dimensional family of tangent sections.

Similarly, for $S=\mathbb{P}^{2}$, any 2-dimensional family of lines satisfies $C_{u}^{2}=m(m-1)+1$ for $m=1$.

Example 4.2. (Abelian surfaces) Let $S$ be an abelian surface and $L$ be a globally generated line bundle on $S$. For any family as in the hypotheses of Theorem 2.1, we have that $C_{u}^{2} \geq m(m-1)+2$. The following is an example where equality is attained.

Let $S$ be an abelian surface with irreducible principal polarization $\Theta$. We assume that $\Theta$ is symmetric. Let $\mu$ denote the endomorphism

$$
\mu: S \ni x \longrightarrow 2 \cdot x \in S
$$

and let $C=\mu^{*}(\Theta)$. Then it is well known (cf. [7, Proposition II.3.6]), that $C \in|4 \Theta|$ and $C$ has multiplicity $m=6$ at the origin. Of course $p_{g}(C)=2$, so that $\operatorname{gon}(C)=2$. We have

$$
32=C^{2}=6 \cdot 5+2 .
$$

Translates of $C$ give a two-dimensional family of curves which are actually algebraically equivalent to $C$ but not linearly equivalent.

Example 4.3. (K3 surfaces) Let $S$ be a $K 3$ surface and $L$ be a globally generated line bundle on $S$. It is well-known that for any positive integer $n$, there exist such $S$ and $L$ with $L^{2}=2 n$. Let $\left\{C_{u}\right\}_{u \in U}$ be any family of curves in $|L|$ satisfying the conditions in Theorem 2.1. Then the theorem yields that

$$
L^{2} \geq m(m-1)+2 .
$$

If $m=1$, this yields that $L^{2} \geq 2$, which is optimal, as $\operatorname{dim}|L|=\frac{1}{2} L^{2}+1=2$ if $L^{2}=2$.

If $m=2$, (8) yields that $L^{2} \geq 4$, which is also optimal. Indeed, if $L^{2}=4$, then $\operatorname{dim}|L|=3$ and for any $x \in S$, we have $\operatorname{dim}\left|L \otimes \mathfrak{m}_{x}^{2}\right| \geq 3-3=0$, so that there is a 2-dimensional family of curves with a point of multiplicity two. A general such curve is nodal of genus two, hence also of gonality 2 .

If $m=3$, (8) yields that $L^{2} \geq 8$, and we do not know if this is optimal. If $L^{2}=10$, however, then $\operatorname{dim}|L|=6$ and for any $x \in S$, we have $\operatorname{dim}\left|L \otimes \mathfrak{m}_{x}^{3}\right| \geq 6-6=0$, so that there is a 2-dimensional family of curves with a point of multiplicity three.

Example 4.4. (Surfaces of general type) Let $S$ be a smooth surface of degree $d \geq 5$ in $\mathbb{P}^{3}$ and consider the family $C_{x}$ of tangent hyperplane sections of $S$, parametrized by $x \in S$. Then a general member of this family is irreducible and has multiplicity $m=2$ at $x$. On the other hand for the canonical divisor $K_{S}$ we have

$$
K_{S}=\mathcal{O}_{S}(d-4)
$$

so that it is $(d-4)$-very ample. This shows that equality can hold in (4) in Theorem 2.1 even if $K_{S}$ is $k$-very ample and not merely birationally $k$-very ample, cf. Lemma 3.2 . 


\section{Applications to Seshadri constants}

In this section we will prove Theorem B, as well as its consequences.

We recall here only very basic notions connected to Seshadri constants. For a systematic introduction to this circle of ideas we refer to chapter 5 in Lazarsfeld's book [8].

Definition 5.1. Let $X$ be a smooth projective variety and $L$ a nef line bundle on $X$. For a fixed point $x \in X$ the real number

$$
\varepsilon(L ; x):=\inf \frac{L \cdot C}{\operatorname{mult}_{x} C}
$$

is the Seshadri constant of $L$ at $x$ (the infimum being taken over all irreducible curves $C$ passing through $x$ ).

For arbitrary line bundles Nakamaye introduced in [9] the notion of moving Seshadri constants $\varepsilon_{\mathrm{mov}}(L ; x)$, see also [5]. If $L$ is nef, then the both notions coincide. If $L$ is big, then the function $\varepsilon_{\text {mov }}(L,-)$ on $S$ assumes its maximal value for $x$ very general, that is, away from a countable union of proper Zariski closed subsets. This was proved by Oguiso [10] for $L$ ample but the proof goes through for $L$ big. This maximal value will be denoted by $\varepsilon(L ; 1)$. We use it in Corollary 5.2 .

If there exists a curve $C \subset X$ actually computing the infimum in the above definition, then we call such a curve a Seshadri curve (for $L$, through $x$ ). It is not known if Seshadri curves exist in general.

If $X=S$ is a surface, then there is an upper bound on Seshadri constants

$$
\varepsilon(L ; x) \leq \sqrt{L^{2}} .
$$

If $\varepsilon(L ; x)$ is strictly less than $\sqrt{L^{2}}$, then there exists a Seshadri curve through $x$. This observation is fundamental for both results presented in this section.

Before proving Theorem B, we need to introduce the following notation. This takes care of bounding the gonality of curves in the family appearing in the proof of Theorem B. We define

(9) $\quad \mu_{S}:=\max \left\{d \mid K_{S}\right.$ is birationally $(d-2)$-very ample or $S$ is birational

to a surface dominating a smooth curve of gonality $d\}$.

In particular, note that $\mu_{S} \geq 2$ if $S$ is irrational and $\mu_{S} \geq 3$ if $\left|K_{S}\right|$ defines a birational map. Also note that $\ell \geq \mu_{S}$, too, by Lemma 3.2, where $\ell$ is as in Theorem 2.1. (We will use this fact in the proof below.)

Proof of Theorem B. First of all (1) implies that for every point $x \in S$ there exists a Seshadri curve $C_{x}$. Such a curve need not to be unique but there are only finitely many of them for every point $x$, see [13, Prop. 1.8]. Since there are only countably many components in the Hilbert scheme of curves in $S$, there must exist a component containing at least a 1-dimensional family $U$ of curves $C_{x}$. If there is more than one such component, we take one for which the dimension of $U$ is maximal. Note, that for curves in $U$ we must have $C_{x}^{2} \geq 0$. Let $m$ denote the multiplicity of a general member of this family in its marked point.

It might happen that $\operatorname{dim} U=1$, but this means that a Seshadri curve $C_{x}$ equals to $C_{y}$ for $y \in C_{x}$ very general. Of course in this case it must be $m=1$, because a 
Seshadri curve is reduced and irreducible. Then the index theorem together with (1) gives

$$
C_{x}^{2} L^{2} \leq\left(L \cdot C_{x}\right)^{2}<\left(1-\frac{1}{4 \mu_{S}}\right) L^{2}<L^{2},
$$

which implies $C_{x}^{2}=0$. It is well known that a moving curve of self-intersection 0 is semi-ample and thus $S$ is fibered as claimed.

So we may assume that $\operatorname{dim} U=2$ and $m \geq 2$. Hence $C_{x}^{2} \geq m(m-1)+\mu_{S}$ by Theorem 2.1 and Lemma 3.2. Revoking again the index theorem we get therefore

$$
\left(m(m-1)+\mu_{S}\right) L^{2} \leq C_{x}^{2} L^{2} \leq\left(C_{x} \cdot L\right)^{2}=m^{2} \varepsilon(L ; 1)^{2}<m^{2}\left(1-\frac{1}{4 \mu_{S}}\right) L^{2} .
$$

It is elementary to observe that the real valued function

$$
f(m)=\frac{m(m-1)+\mu_{S}}{m^{2}}
$$

for $m \geq 2$ attains the minimal value at $m=2 \mu_{S}$ and $f\left(2 \mu_{S}\right)=1-\frac{1}{4 \mu_{S}}$, and we arrive at a contradiction to (10). This concludes the proof of Theorem B.

As a consequence, we obtain the following result yielding lower bounds on the Seshadri constant of the canonical bundle at very general points, which improves and generalizes [3, Thms. 2 and 3].

Corollary 5.2. Let $S$ be a minimal smooth projective surface of general type, i.e., such that $K_{S}$ is big and nef. Then either $\varepsilon\left(K_{S} ; 1\right) \geq \sqrt{\frac{7}{8} K_{S}^{2}}$ or $S$ is fibered by Seshadri curves of $K_{S}$-degree $\varepsilon\left(K_{S} ; 1\right) \geq 2$.

If furthermore $K_{S}$ is birationally $k$-very ample, for an integer $k \geq 1$, then either $\varepsilon\left(K_{S} ; 1\right) \geq \sqrt{\left(1-\frac{1}{4(k+2)}\right) K_{S}^{2}}$ or $S$ is fibered by Seshadri curves of $K_{S}$-degree $\varepsilon\left(K_{S} ; 1\right) \geq 4 k$.

Note that the hypothesis that $S$ is minimal i.e., $K_{S}$ is nef is not restrictive, since if $\widetilde{S} \rightarrow S$ is a birational morphism, then one easily sees that $\varepsilon_{\operatorname{mov}}\left(K_{\widetilde{S}} ; 1\right)=\varepsilon\left(K_{S} ; 1\right)$.

Proof. If $K_{S}$ is big and nef, but not birationally 1-very ample, set $k=0$. Then, under the assumptions of the corollary, we have $\mu_{S} \geq k+2$. By Theorem B, if $\varepsilon\left(K_{S} ; 1\right)<\sqrt{\left(1-\frac{1}{4(k+2)}\right) K_{S}^{2}}$, then $S$ is fibered by (smooth) Seshadri curves $C$ of $K_{S}$-degree $C \cdot K_{S}=\varepsilon\left(K_{S} ; 1\right)$. Furthermore, we must have $C \cdot K_{S} \geq 2$ by adjunction, as $C^{2}=0$, and the first assertion follows.

If $K_{S}$ is birationally $k$-very ample for $k \geq 1$, then the general curve $C$ must have gonality $\operatorname{gon}(C) \geq k+2$ by Lemma $3.2(\mathrm{a})$. At the same time, by the existence theorem of special divisors of Kempf-Kleiman-Laksov (see e.g. [1, V.1.1]), a smooth curve $C$ of genus $p_{a}(C)$ carries a pencil of arbitrary degree $d \geq \frac{p_{a}(C)+2}{2}$, whence $\operatorname{gon}(C) \leq \frac{p_{a}(C)+3}{2}$. Moreover, by adjunction we have $K_{S} \cdot C=2 p_{a}(C)-2$. Hence

$$
K_{S} \cdot C=2 p_{a}(C)-2 \geq 2(2 \operatorname{gon}(C)-3)-2 \geq 2(2(k+2)-3)-2=4 k,
$$

and the second assertion follows.

Our next and last result parallels [4, Theorem] and [14, Theorem 1]. The arguments are similar to those in the proof of Theorem B, but it seems that the result itself is of independent interest and is not a straightforward corollary of Theorem B. Note that for $\mu_{S}=1$, we retrieve (in practice) Xu's result. 
Proposition 5.3. Let $S$ be a smooth surface and $L$ a big and nef line bundle on $S$. Assume that, for a given integer $a \geq 1$, we have that either

or

$$
L^{2}>\frac{4 \mu_{S} a^{2}-4 a+4}{4 \mu_{S}-1},
$$

Then, either

$$
L^{2}=\frac{4 \mu_{S} a^{2}-4 a+4}{4 \mu_{S}-1} \text { and } \frac{L^{2}-2 a}{2\left(L^{2}-a^{2}\right)} \notin \mathbb{Z} .
$$

(i) $\varepsilon(L ; 1) \geq a$, or

(ii) $S$ is fibered in Seshadri curves (of L-degree $<a$ ).

Remark 5.4. The case $\frac{L^{2}-2 a}{2\left(L^{2}-a^{2}\right)} \notin \mathbb{Z}$ also includes the case $L^{2}=a^{2}$. However, if $L^{2} \geq \frac{4 \mu_{S} a^{2}-4 a+4}{4 \mu_{S}-1}$, it is easily seen that $L^{2} \geq a^{2}$, with equality only if $L^{2}=4$ and $a=2$. The proposition holds in this special case.

Proof. As in the proof of $[4$, Thm.] or [14, Theorem 1], the set

$$
V:=\left\{(C, x) \mid x \in C \subset S \text { a reduced, irreducible curve, } \operatorname{mult}_{x}(C)>\frac{C \cdot L}{a}\right\}
$$

consists of at most countably many families. (This conclusion also holds when $L$ is big and nef but not ample.)

Assume that we are not in case (i) of the proposition, that is, assume that $\varepsilon(L ; 1)<$ a. Then $\operatorname{dim} V \geq 2$.

By our assumptions we have $L^{2} \geq a^{2}$, cf. Remark 5.4. Therefore, the index theorem yields, for any curve $C \subset S$, that

$$
C \cdot L \geq \sqrt{L^{2} \cdot C^{2}} \geq a \sqrt{C^{2}} .
$$

It follows that if $C \cdot L<a$ for a curve $C \subset S$ moving in a nontrivial algebraic family, then $C^{2}=0$, so that we are in case (ii) of the proposition. Otherwise we must have a nonempty subset of dimension $\geq 2$ of $V$ consisting of pairs $(C, x)$ with mult $_{x}(C)>1$. Since each curve in question is reduced, we can find a two-dimensional irreducible scheme $U \subset$ Hilb $S$ parametrizing curves in $V$ each with mult ${ }_{x} C>1$. Letting $m$ be the multiplicity of the general curve in this family and $C$ be the algebraic equivalence class, we have $C^{2} \geq m(m-1)+\mu_{S}$ by Theorem 2.1 and Lemma 3.2.

As $m>\frac{C . L}{a}$, we have $C \cdot L \leq m a-1$, whence by the index theorem,

$$
(m a-1)^{2} \geq(C \cdot L)^{2} \geq C^{2} L^{2} \geq\left(m^{2}-m+\mu_{S}\right) L^{2},
$$

so that

$$
\left(L^{2}-a^{2}\right) m^{2}+\left(2 a-L^{2}\right) m+\left(L^{2} \mu_{S}-1\right) \leq 0 .
$$

If $L^{2} \leq a^{2}$, then $L^{2}=a^{2}=4$ by Remark 5.4 , so that (11) yields $4 \mu_{S} \leq 1$, a contradiction. Hence $L^{2}>a^{2}$, so that the function

$$
f(x):=\left(L^{2}-a^{2}\right) x^{2}+\left(2 a-L^{2}\right) x+\left(L^{2} \mu_{S}-1\right)
$$

attains its minimal value $f_{\text {min }}$ at $x=\frac{L^{2}-2 a}{2\left(L^{2}-a^{2}\right)}$ and

$$
f_{\text {min }}=\frac{L^{2}}{4\left(L^{2}-a^{2}\right)}\left[\left(4 \mu_{S}-1\right) L^{2}-4\left(\mu_{S} a^{2}-a+1\right)\right] .
$$


By our assumptions on $L^{2}$ it follows that $f(x) \geq 0$ for all $x$ and that $f(x)=0$ only

if $L^{2}=\frac{4 \mu_{S} a^{2}-4 a+4}{4 \mu_{S}-1}$ and then at the point $x=\frac{L^{2}-2 a}{2\left(L^{2}-a^{2}\right)}$, which is not an integer by assumption. Therefore $f(m)>0$ for all $m \in \mathbb{Z}$, contradicting (11).

\section{Acknowledgements}

This work has been partially supported by the SFB/TR 45 "Periods, moduli spaces and arithmetic of algebraic varieties". The second and the third author were partially supported by a MNiSW grant N N201 388834. The authors thank E. Sernesi and F. Flamini for helpful correspondence. Furthermore, the authors would like to thank the referee for careful reading of the manuscript and helpful comments and remarks.

\section{References}

[1] E. Arbarello, M. Cornalba, P. Griffiths, and J. Harris, Geometry of Algebraic Curves, vol. I, Springer-Verlag (1985).

[2] F. Bastianelli, Remarks on the nef cone on symmetric products of curves, to appear in Manuscripta Mathematica.

[3] T. Bauer and T. Szemberg, Seshadri constants on surfaces of general type, Manuscr. Math. 126 (2008) $167-175$.

[4] L. Ein and R. Lazarsfeld, Seshadri constants on smooth surfaces, Astérisque 218 (1993) 177186.

[5] L. Ein, R. Lazarsfeld, M. Mustata, M. Nakamaye, and M. Popa, Restricted volumes and base loci of linear series, to appear in Amer. J. Math.

[6] A. L. Knutsen, On kth order embeddings of K3 surfaces and Enriques surfaces, Manuscr. Math. 104 (2001) 211-237.

[7] H. Lange and C. Birkenhake, Complex Abelian Varieties, Vol. 302 of Grundlehren der mathematischen Wissenschaften, Springer-Verlag (1992).

[8] R. Lazarsfeld, Positivity in Algebraic Geometry I, Springer-Verlag (2004).

[9] M. Nakamaye, Base loci of linear series are numerically determined, Trans. Amer. Math. Soc. 355 (2002) 551-566.

[10] K. Oguiso, Seshadri constants in a family of surfaces, Math. Ann. 323 (2002) 625-631.

[11] E. Sernesi, Deformations of algebraic schemes, Vol. 334 of Grundlehren der Mathematischen Wissenschaften, Springer-Verlag (2006).

[12] W. Syzdek and T. Szemberg, Seshadri fibrations of algebraic surfaces, to appear in: Math. Nachr.

[13] T. Szemberg, Global and local positivity of line bundles, Habilitationsschrift, Essen 2001.

[14] G. Xu, Ample line bundles on smooth surfaces, J. Reine Angew. Math. 469 (1995) 199-209.

Department of Mathematics, University of Bergen, Johannes Brunsgate 12, 5008 Bergen, NORWAY

E-mail address: andreas.knutsen@math.uib.no

Instytut Matematyki, Uniwersytet Pedagogiczny, Podchorażych 2, PL-30-084 Kraków, POLAND

Current address: Mathematisches Institut, Universität Duisburg-Essen, 45117 Essen, Germany

E-mail address: wioletta.syzdek@uni-due.de

Instytut Matematyki, Uniwersytet Pedagogiczny, Podchorażych 2, PL-30-084 Kraków, POLAND

Current address: Instytut Matematyczny, PAN, ul. Śniadeckich 8, PL-00-956 Warszawa, Poland

E-mail address: szemberg@ap.krakow.pl 